\title{
Die Narrativität fiktionaler und realer Konversationspolitik: Diderot und die öffentliche Deliberation gesamtgesellschaftlicher Probleme
}

In meiner komparatistischen Studie Biographische Fiktionen: Das Paradigma Denis Diderot im interkulturellen Vergleich (1765-2015) wurde deutlich, dass Diderot als literarische Hauptfigur über die Jahrhunderte hinweg eher apolitisch rezipiert wurde. ${ }^{1}$ Trotz der Tatsache, dass einige Experten der Aufklärung des achtzehnten Jahrhunderts - wie beispielsweise Anthony Strugnell oder Yves Benot - Diderot als eigentlich den politischsten aller Schriftsteller der französischen Aufklärung bezeichnet haben, ${ }^{2}$ ist dieses Ergebnis jedoch keineswegs außergewöhnlich. Biopics, fiktionalbiographische Romane und Theaterstücke über Schriftsteller, Künstler und Philosophen konzentrieren sich generell eher auf das innere Seelenleben oder das ausgelebte beziehungsweise unterdrückte Liebesleben historischer Persönlichkeiten und weniger auf politische Ereignisse, Überzeugungen und Kontroversen. Umso interessanter war es dann aber auch festzustellen, dass die mir vorliegenden englischsprachigen Texte Diderot fast ausschließlich als einen weitsichtigen politischen Intellektuellen rezipiert und fiktionalisiert haben. ${ }^{3}$ So erschien beispielsweise 1980 ein fiktives Interview

1 Heidi Denzel de Tirado: Biographische Fiktionen im interkulturellen Vergleich. Das Paradigma Denis Diderot (1765-2005), Würzburg 2008.

2 »Diderot [...] reste néanmoins l'écrivain le plus politique des Lumières [...] Ni Voltaire, trop épris de son rôle personnel dans les affaires publiques, ni Rousseau, dont l'inspiration politique est viciée par son orgueil persécuté, ne nous proposent en leur personne l'image de l'homme nouveau préparant les temps nouveaux, tel qu'il se dégagera des pages que rédigera Diderot après son départ de la cour impériale.« Anthony Strugnell: [Art.] Politique, in: Dictionnaire de Diderot, hg. von Roland Mortier und Raymond Trousson, Paris 1999, S. 413. Siehe auch: Hans-Jürgen Lüsebrink, Manfred Tietz: Lectures de Raynal, Oxford 1991; Hans-Jürgen Lüsebrink, Anthony Strugnell: L'Histoire des deux Indes: réécriture et polygraphie, Oxford 1995. Ebenso zum Thema: Lester G. Crocker: Diderot as Political Philosopher, in: Revue internationale de philosophie, 148 (1984), S. 120-139; Paul Vernière: Diderot et les contradictions de sa pensée politique, in: Revue des sciences morales et politiques, 139 (1984), S. 269-285. Diese Tendenz der englischsprachigen Texte, Diderot als politischen Denker darzustellen, könnte zurückzuführen sein auf Anthony Strugnell: Diderot's Politics: A Study of the Evolution of Diderot's Political Thought after the Encyclopedie, The Hague 1973. 
mit Diderot in der New York Times unter dem Titel »For Liberals to Stay in the 80's Mainstream: A Rephrasing of Theories Was in Order the Estimable Diderot >Told a ’Reporter«. Darin wurde Diderot als »Parteivorsitzender der Aufklärungspartei« bezeichnet, der nach seiner Einschätzung der gegenwärtigen politischen Lage der USA unter Ronald Reagan befragt wird. ${ }^{4}$ Schon ein Jahr später erschien Edward T. Sanders politisches Pamphlet The Z-D Generation, das Diderot als Kritiker und »Geist« des Widerstands gegen den Staat als totalitäres Organ der Gesellschaft präsentiert, zelebriert und radikalisiert. ${ }^{5} \mathrm{Im}$ Jahr 2000 tauchte Diderot schließlich als politischer Berater der russischen Zarin Katharina der Großen in Malcolm Bradburys Roman To the Hermitage auf. ${ }^{6}$ Dieser Text reflektiert wie kein anderer Diderots politisches Genie und seine virtuose Expertise, seine politischen Ansichten in die verschiedensten Formen und Gattungen einzuweben. Bradbury bezieht sich hier oft auf Diderots Werke, die heute allgemein als seine politischsten Texte angesehen werden, und zitiert mehrmals wortwörtlich aus den Mémoires pour Catherine II, die auch unter dem Namen Mélanges philosophiques, historiques, etc., pour Catherine II bekannt sind, Les Observations sur le Nakaz, seinen Beiträgen für die Histoire des deux Indes und natürlich seinem Supplément aux Voyages de Bougainville. Obwohl Diderots englischsprachige Rezipienten aus dem zwanzigsten Jahrhundert nicht verschiedener sein könnten und ihre biographischen Fiktionen unterschiedlichen Textformen angehören, haben sie doch eine Gemeinsamkeit: Sie folgen einem dialogischen Prinzip deliberativer Konversationen und hinterfragen bestehende politische Institutionen, Instanzen und Praktiken. Diese Form von »Konversationspolitik« ist auch in den deutschsprachigen biographischen Fiktionen zeitweise

4 Gerald Weissman: For Liberals to Stay in the 80's Mainstream, A Rephrasing of Theories Was in Order The Estimable Diderot »Told« a »Reporter«, in: New York Times, 2. Dezember, 1980, S. A19. Hier ist der Autor Gerald Weissman besonders darauf bedacht, Diderots progressive Ansichten mit der überholten amerikanischen Politik der 1980er zu kontrastieren.

5 Edward Sanders: The Z-D Generation, Barrytown 1981. In diesem Text kämpft Diderot gemeinsam mit Emile Zola als politisch engagierter Intellektueller gegen Unrecht und Korruption innerhalb des Polizeistaats Frankreich. Hier wird Diderot als Kritiker und »Geist« des Widerstands gegen den Staat als totalitäres Organ der Gesellschaft repräsentiert, zelebriert und radikalisiert.

6 Malcolm Bradbury: To the Hermitage, Basingstoke/Oxford 2000. Bradbury vergleicht in seinem Roman politische Ereignisse der Gegenwart mit der Zeit des Aufenthalts von Diderot in Sankt Petersburg, zum Beispiel die Anti-Perestroika-Bewegungen in Russland der 1990er Jahre mit der Pugatschew-Rebellion im achtzehnten Jahrhundert. 
präsent, besonders in Leopold von Sacher-Masochs Novelle Diderot in Sankt Petersburg (1873) und Peter Pranges Roman Die Philosophin (2005), die im Folgenden genauer analysiert werden.

Diderots produktive Rezeption in Deutschland und die Fiktionalisierung seiner "Konversationspolitik"

Im Gegensatz zum englischsprachigen Raum hat sich die produktive Rezeption Diderots in deutschsprachigen Fiktionen weitaus weniger auf seine Rolle als politischer Visionär konzentriert. Zwar hat Hans Magnus Enzensberger - einer der politischsten Literaten Deutschlands und Preisträger des Literaturpreises »Das politische Buch« zur Förderung wichtiger politischer Literatur - Diderot mit »viel Vergnügen und Interesse rezipiert und ihm sogar eine Monographie mit dem Titel Diderots Schatten: Unterhaltungen, Szenen, Essays (1994) gewidmet, doch konzentriert sich Enzensberger in diesem Buch eher auf Diderots Rolle als erster »Intellektueller« überhaupt:

Diderot hat die Rolle des Intellektuellen nicht nur erfunden. Er wendete sie hin und her, und er sah, dass sie nicht nur schwierig, sondern auch prekär, nicht nur undankbar, sondern auch eine Quelle überraschender Paradoxe war. Sie warf gravierende moralische Probleme auf und entbehrte nicht einer abgründigen Komik. ${ }^{7}$

Die spielerische Art Diderots, die Intellektuellenrolle in Dialogen zu problematisieren und zu hinterfragen, ist nicht nur in Enzensbergers fiktivem Radiointerview Diderot und das dunkle Ei (1978) präsent, sondern auch in anderen deutschen Texten wie Leopold von Sacher-Masochs Novelle Diderot in Sankt Petersburg (1873), Peter Pranges Roman Die Philosophin (2005) und Michael Krügers Gedicht Diderots Katze (1978). In Krügers Gedicht erscheint Diderot gemeinsam mit einer Katze als ideales Harmoniemodell, in dem der Philosoph für einen »aufgeklärten Rationalismus « und die Katze für einen »instinktiven Subjektivismus« stehen. Diderot erscheint hier aber keineswegs als politischer Revolutionär oder Visionär. Von der Arbeit an der Enzyklopädie müde und

7 Hans Magnus Enzensberger: Diderots Schatten. Unterhaltungen, Szenen, Essays, Frankfurt 1994, S. 385. 
verdrossen, beklagt er hier »meckernd« das »Altern einer Illusion«. Die anthropomorphisierte und hochintelligente Katze, die spontan »eine Abhandlung über den Einfluss des Klimas aufs milchige Fensterglas schreibt (während Diderot vor dem Fenster die Revolution beobachtet, ihre vorsichtigen Schritte)«, dient hier als Diderots Gesprächspartner und Kontrastfigur und bringt ihn zu der desillusionierenden Erkenntnis, »dass die Philosophie auch nur eine Anleitung zum Sterben « ist. $^{8}$ In Enzensbergers Diderot und das dunkle Ei trifft Diderot auf einen Reporter aus dem zwanzigsten Jahrhundert. In diesem anachronistischen Dialog bekommen Diderots politische Ansichten schon etwas mehr Aufmerksamkeit und der Reporter erklärt ihm, dass die Deutschen ihn in der Zukunft, in 200 Jahren, besonders dafür schätzen werden, dass er sich stets für »die Schwachen«, »das Volk« und »die Rechte der unterworfenen Nationen« eingesetzt habe und sogar dafür ins Gefängnis gegangen sei. Der französische Aufklärer zeigt sich daraufhin aber revolutionskritisch, sagt »schlimme « Zeiten voraus und prophezeit, dass es eines Tages »in Europa von Helfern nur so wimmeln« werde: »Jeder wird sich berufen fühlen, gegen die Missstände der politischen und moralischen Welt anzutreten [...] Wohin man blickt, wird man Scharen von Philanthropen, Pädagogen, Vormündern, Treuhändern antreffen, die alle nur das Beste wollen. ${ }^{9}$

Auch in Sacher-Masochs Novelle Diderot in Sankt Petersburg erscheint Diderot nicht als Prometheus der Aufklärung, der politische oder utopische Lösungen ersinnt. Sacher-Masoch ist vielmehr - ganz im Sinne von Katie Trumpeners Lesart von Diderots Bijoux Indiscrets - an dem impliziten Thema von Möglichkeiten und Limitationen politischer Reden und Dialoge interessiert. ${ }^{10}$ Anstatt explizit verbalisierter politischer Auseinandersetzungen zeigt Sacher-Masoch nach Diderot'scher Art auf, wie alltägliche Konversationen von politischen Verhältnissen geprägt sind und wie sie Voraussetzungen für ein politisches Leben werden können. In diesem Sinne werde ich mich in der folgenden Analyse der kaum bekannten und vergriffenen Novelle Diderot in Sankt

8 Michael Krüger: Diderots Katze. Fotografiert von Gabriele Lorenzer, in: Diderots Katze. Gedichte, München/Wien 1978, S. 69-72, hier S. 72.

9 Enzensberger: Diderots Schatten (wie Anm. 7), S. 39.

10 Katie Trumpener: The Politics of Conversation. Denis Diderot, Elio Vittorini, Manuel Puig, Masaki Kobayashi, Vasily Grossman, in: Scheherazade's Children. Global Encounters with the Arabian Nights, hg. von Philip F. Kennedy und Marina Warner, New York 2013, S. 218-242, hier S. 225. 
Petersburg von Leopold von Sacher-Masoch auf die Dynamik von Dialogen und Konversationen als Grundlagen politischen Lebens konzentrieren. In diesem Zusammenhang werde ich mich auf das dialogische Prinzip deliberativer Demokratie nach Jürgen Habermas beziehen, um Diderots Intellektuellenrolle und seine »Konversationspolitik« in Russland zu erörtern.

Leopold von Sacher-Masochs Novelle Diderot in Sankt Petersburg (1873) und das dialogische Prinzip deliberativer Demokratie

Der Plot der Novelle von Sacher-Masochs Diderot in Sankt Petersburg ist schnell erzählt: Sacher-Masoch lässt Diderot in der Sankt Petersburger Akademie der Wissenschaften einen Vortrag über »die Verwandtschaft des Menschen mit den Tieren und seine Abstammung von dem Affen « halten. ${ }^{11}$ Während diese Hypothese allgemein enthusiastisch von seinem russischen Publikum aufgenommen wird, wagt jedoch der Zoodirektor und Tierpräparator Lagetschnikoff Diderots Behauptung zu hinterfragen und fordert Beweise. Diderot wehrt diesen Einwand erstaunt ab und ist überrascht, dass man in Russland tatsächlich noch nie von den real existierenden sprechenden Affen in Madagaskar gehört haben will. Die russische Zarin, Katharina die Große, will daraufhin sofort ein Exemplar dieser Spezies der sprechenden Affen, und Diderot - in Beweisnot geraten - verkleidet sich auf Ratschlag der Fürstin Daschkoff selbst als Affe, um die Zarin zu unterhalten. Katharina trifft sich nun täglich mit ihrem sprechenden »Kammeraffen« und ist vorübergehend durchaus begeistert. Als der »Affe« aber keine neuen Kunststücke mehr lernen will, vermacht sie den als Affe verkleideten Philosophen kurzerhand dem Zoodirektor Lagetschnikoff, der ihn dort mit Peitsche und Folter dressieren möchte. Als Lagetschnikoff den ungelehrigen »Affen« ausstopfen will, gesteht ihm Diderot in seiner Angst seine wahre Identität. Doch Lagetschnikoff zeigt keine Gnade und verkündet: "Ich stopfe Sie aus, gerade so, wie Sie mich ausgestopft hätten, wenn Sie Kaiser von Russland geworden wären. ${ }^{12}$ Diderot muss

11 Leopold von Sacher-Masoch: Diderot in Sankt Petersburg, hg. von Dieter Bandhauer, Wien 1987 [1875], S. 5-58, hier S. 17.

12 Ebd., S. 57. 
daraufhin auf Aufforderung seines Peinigers Lagetschnikoff seinem Atheismus abschwören und überlebt schließlich nur aufgrund des Eingreifens der Fürstin Daschkoff.

Im Gegensatz zu Diderots Liebe für Diskussionen und Auseinandersetzungen, die er stets besonders im wissenschaftlichen Bereich an den Tag gelegt hatte, folgt Sacher-Masoch hier keineswegs seinem Vorbild Diderot und dessen geliebter sokratischen Methode, die so viele seiner Werke auszeichnet. Anstatt den fiktiven Gesprächspartner Lagetschnikoff und die Leser der Novelle durch geeignete Fragen zu überzeugen und zu einer gewünschten Erkenntnis zu bringen, findet hier kein mäeutischer Dialog statt: »Ich erstaune, dass ein Naturforscher wie Sie nicht weiß, dass es sprechende Affen gibt «, ${ }^{13}$ - heißt es bei Sacher-Masoch ganz einfach.

Ohne zu vertieft auf das Konzept der deliberativen Demokratie einzugehen, erscheint Diderots Rede in der Akademie nach Jürgen Habermas auf den ersten Blick wie eine »ideale Sprechsituation", in der in einem machtfreien Diskurs durch Austausch von Argumenten Verständigung und Konsens erzielt werden können. ${ }^{14}$ Diderots Rede ist ein Erfolg, er wird also - trotz seiner kühnen These der Evolution, die noch 100 Jahre später, zur Zeit der Publikation der Novelle, durchaus kontrovers war - von seinem Publikum bewundert und geschätzt. ${ }^{15}$ Den Grundlagen diskursiver Politik im Habermas'schen Sinne folgend, nimmt der Tierpräparator Lagetschnikoff daraufhin sein Recht in Anspruch, Diderots Autorität in Frage zu stellen und dessen Behauptung zu problematisieren. Doch Sacher-Masochs Diderot respektiert seinen Kritiker keineswegs als »gleichwertiges Subjekt«. Stattdessen bezichtigt er seinen Gesprächspartner nicht nur der Ignoranz, die man allgemein auf die Ahnungslosigkeit des russischen Volkes übertragen könnte, sondern stützt sich insgesamt in seiner Argumentation auf äußerst unlautere Mittel. Damit verletzt er das Habermas'sche Diskursprinzip als

13 Ebd., S. 18.

14 Claudia Landwehr: Facilitating Deliberation. The Role of Impartial Intermediaries in Deliberative Mini-publics, in: Deliberative Mini-publics. Involving Citizens in the Democratic Process, hg. von Kimmo Grönlund, André Bächtiger und Maija Setälä, Colchester 2014, S. 77-92, hier S. 77.

15 Hier ist zu bemerken, dass Diderot tatsächlich eine Rede in der Akademie der Wissenschaften gehalten hat. Darin ging es aber noch nicht um die Evolutionstheorie, die in einigen seiner Texte zwar durchaus schon angedeutet wird, aber noch keineswegs explizit ausformuliert ist. Siehe hierzu Denzel de Tirado: Biographische Fiktionen (wie Anm. 1), S. $307 f$. 
Grundlage des Moral- und des Demokratieprinzips. Diderots Lüge der Existenz einer Spezies von sprechenden Affen ist damit weder »vernünftig« und »aufgeschlossen«, noch »wahrhaftig« und »empathisch«, um nur ein paar der Anforderungen an Diskursbeteiligte einer deliberativen Demokratie zu nennen. ${ }^{16}$

Doch diese explizite Verletzung der Diskursregeln rechtfertigt natürlich keineswegs die heftige Reaktion des russischen Zoodirektors, der Diderot am Ende töten und ausstopfen will. Dies ist nicht nur auf die Kontroverse zwischen Religion, Kreationismus und Darwinismus zurückzuführen. Hier geht es - ganz im Sinne der Diderot'schen Rede in dieser Novelle - vielmehr um »the survival of the fittest«. In unserer Novelle lässt die Gräfin Daschkoff den Zoodirektor nämlich anfangs glauben, dass Diderot ihm seine offene Kritik und seine Frage nicht verziehen habe und ihn deshalb zerstören wolle:

Armer, armer Freund, Sie sind verloren...Diderot hat der Kaiserin eine Liebeserklärung gemacht...Denken Sie sich nun Diderot als Zaren und Sie als seinen Untertan, er ist imstande und lässt Sie an Stelle des redenden Affen mit dem Sie ihm das Leben so sauer gemacht haben, für das Museum ausstopfen. ${ }^{17}$

Diese absurd klingende Drohung war im 19. Jahrhundert nicht ganz so abwegig, und ausgestopfte Menschen in Museen waren keineswegs eine Erfindung Sacher-Masochs. Der »Kammermohr« des Fürsten Johann Georg Christian von Lobkowitz, Angelo Soliman, wurde beispielsweise nach seinem Tod tatsächlich ausgestopft und bis $1806 \mathrm{im}$ Naturalienkabinett des österreichischen Kaisers ausgestellt. ${ }^{18}$ Überdies war Lagetschnikoffs Angst, Diderot könne sich bei Katharina über ihn beschweren, nicht ganz von der Hand zu weisen, weil dieser als Kulturbeauftragter im französisch-russischen Austausch durchaus die

${ }^{16}$ Für eine genauere Definition der Habermas'schen Diksursregeln, siehe Markus Spörndli: Diskurs und Entscheidung. Eine empirische Analyse kommunikativen Handelns im deutschen Vermittlungsausschuss, Wiesbaden 2013, S. 15-18.

17 Sacher-Masoch: Diderot in Sankt Petersburg (wie Anm. 11), S. 36.

18 Für mehr Details über das Leben des »Kammermohrs« Angelo Soliman, eine Analyse rassenpolitischer Aspekte und Parallelen zu Völkerschauen bei Sacher-Masoch, siehe Heidi Denzel de Tirado: Von szivilisierten Affen< und `barbarischen Menschen<. Der 'mumifizierte Kammermohr Angelo Soliman, Kafka und Sacher-Masoch, in: Texturen des Barbarischen. Exemplarische Studien zu einem Grenzbegriff der Kultur, hg. von Carla Dauven von Knippenberg, Christian Moser und Daniel Wendt, Heidelberg 2013, S. 95-113. 
Aufgabe gehabt hatte, junge russische Intellektuelle zu beaufsichtigen und Katharina über deren Benehmen zu informieren. ${ }^{19}$

Doch warum lässt sich Diderot im wahrsten Sinne des Wortes von der Zarin zum "Affen « machen? Hier erscheint es mir wichtig herauszustellen, dass es bei Sacher-Masoch die Gräfin Daschkoff ist, die ihn zu dieser Maskerade überreden kann, weil sie von Anfang an vorgibt, dass nur er Einfluss auf die Zarin nehmen könne:

Die Kaiserin, die absolute Herrin von fünfzig Millionen Sklaven, langweilt sich wieder einmal, wissen Sie, was das heißt, eine Zarin langweilt sich? Das bedeutet so viel als: Russland zittert und erwartet von Ihnen seine Befreiung von dem kaiserlichen Zorne. Allen Ernstes, Sie sind der Einzige...dem wir alle und vor allem die Kaiserin selbst es zutrauen, dass Sie deren böse Laune...zerstreuen... ${ }^{20}$

Überdies lässt die Gräfin Daschkoff Diderot glauben, die Zarin sei in ihn verliebt und wolle ihn heiraten, während sie Katharina der Großen erklärt, dass sie einen Liebesbrief von Diderot an sie gefunden habe. Nun hat Diderot in Wirklichkeit natürlich nie geglaubt, dass er vielleicht Zar von Russland werden könnte, aber er hatte doch Hoffnungen, die Philosophie auf den russischen Thron zu bringen. Wie Platon war Diderot überzeugt, dass die Herrscher zuerst aufgeklärt werden

19 Wie Sacher-Masoch sah auch Diderot den Osten als "para-kolonialen Raum atavistischer Traditionen am Rande der Zivilisation«, um Stephanie Weissman zu zitieren. Und Sacher-Masochs Geschichten, besonders seine galizischen Geschichten, zeigen wie Diderots Supplément au Voyage de Bougainville, dass Zivilisation oft nur eine Maske für gewaltsame Eroberungen darstellt. Wie Sacher-Masoch im Falle des Habsburgischen Reiches, wehrte sich auch Diderot gegen die Idee, die französische Kultur im Ausland zu indoktrinieren. Im Plan d'une Université postuliert er, dass jedes Land seine eigene Kultur entwickeln sollte und nicht einfach die schlechte Kopie des französischen Vorbilds sein sollte. In diesem Sinne ist es interessant festzustellen, dass Sacher-Masoch Diderots Feind, den Zoodirektor Lagetschnikoff, nicht nur als einen »Wilden" oder »Barbaren« darstellt, sondern auch als einen ehemaligen Stipendiaten, der an ein paar deutschen Universitäten studiert und ein Jahr in Paris verbracht hat. Dort hat er, laut Sacher-Masoch alles, was er vorher so eminent gekonnt hatte, verlernt und im Gegenzug außer ein paar »Phrasen der Pariser Philosophen...nichts Ordentliches « gelernt. Siehe hierzu Sacher-Masoch, Diderot in Sankt Petersburg (wie Anm. 11), S. 10. Diese Beschreibung Lagetschnikoffs erinnern durchaus an Äußerungen Diderots über russische Stipendiaten: »[J]e crois que les voyages, comme les font aujourd'hui nos jeunes seigneurs, corrompent plus de jeunes gens qu'ils n'en instruisent." $\mathrm{Zu}$ einer genaueren Analyse von kultur- und bildungspolitischen Aspekten, siehe Denzel de Tirado: Biographische Fiktionen (wie Anm. 1), S. 319-321.

20 Sacher-Masoch: Diderot in Sankt Petersburg (wie Anm. 11), S. 6. 
sollten, ${ }^{21}$ und Katharina hatte sich als sehr offen, bescheiden und innovativ gezeigt und die philosophes mit ihrem Gesetzesentwurf - auf russisch kurz »нака́зъ« (Nakaz) genannt - schwer beeindruckt. Außerdem schien zur Zeit von Diderots Russlandreise der Absolutismus im Westen die Oberhand zu gewinnen, ${ }^{22}$ und nur Russland zeigte sich weiterhin wie ein Paradebeispiel für die Möglichkeit, ein Land zu zivilisieren. ${ }^{23}$ Nach dem Vorbild Platons und dessen Unterredungen mit dem Tyrannen Dionysios von Syrakus, traf sich Diderot daher täglich mit Katharina der Großen, um der Zarin die Ideale der Aufklärung näherzubringen. Während Platon im "philosophischen Exkurs« seines Siebten Briefs klarmacht, dass es sich bei seinen Zusammenkünften mit dem berüchtigten Gewaltherrscher nicht um Lektionen handle und die Erkenntnisse ihrer Gespräche nie schriftlich niedergelegt werden sollten, sind Diderots Aufzeichnungen seiner Konversationen mit der Zarin in den Mémoires pour Catherine 1899 erschienen - und damit Sacher-Masoch höchstwahrscheinlich unbekannt. Ob es sich bei diesen Dialogen tatsächlich um wahre Aufzeichnungen der Gespräche zwischen der Zarin und dem Philosophen oder um autobiographische Fiktionen handelt, sei hier dahingestellt. Ich möchte vielmehr auf die Vielseitigkeit der Diderot'schen Rollen in seinen eloquenten Gesprächen mit der Zarin hinweisen. So gibt er sich beispielsweise als »Träu-

21 Siehe z.B.: Denis Diderot: Pages contre un tyran, in: Euvres politiques, hg. von Paul Vernière, Paris 1963, S. 142. Hier sei noch auf Friedrich Melchior Grimms Correspondance littéraire hingewiesen, für die Diderot häufig schrieb. Grimm hoffte auf eine Gemeinschaft von Philosophen und aufgeklärten Prinzen und wandte sich daher mit seiner alle zwei Wochen erscheinenden Zeitschrift, der Correspondance littéraire, an eine kleine Gruppe von auserwählten Rezipienten aus gehobenen Kreisen, die er für fähig hielt, dem »esprit humain« folgen zu können und sich von Ideen der Lumières begeistern lassen zu können. Die Correspondance littéraire schien ihm ein wichtiges Instrument der Aufklärung, und sowohl der ausschließlich auf souveräne Fürsten eingeschränkte Empfängerkreis seiner geheimen Korrespondenz als auch die Auswahl der Themenbeiträge zeugen von einer durchaus didaktischen Intention, eine erlesene Elite für die Ideen der Aufklärung zu gewinnen.

221771 lösten Louis XV und Maupeou die Parlamente auf. In Deutschland hatte sich der »Philosophenkönig « Friedrich zum Despoten gewandelt und in Schweden änderte Gustav III 1772 die Verfassung und stärkte damit seine eigene Exekutivgewalt und schwächte die seit über 50 Jahren relativ starke Rolle des Riksdag. Sogar in England, der von Montesquieu so hoch gelobten parlamentarischen Monarchie, herrschte seit Jahren Chaos, Korruption und Instabilität im Parlament, und George III zögerte nicht mehr, seine Exekutivgewalt für seine Zwecke einzusetzen.

23 Siehe hierzu Marc Raeff: The Enlightenment in Russia and Russian Thought in the Enlightenment, in: The Eighteenth Century in Russia, hg. von J.G. Garrard, Oxford 1973, S. 25-47, hier S. 46 f. 
mer«, als ein vor sich hinbrabbelndes Kind, als begeisterter Feriengast, als Pazifist, als Apostel, als liebevoller Vater, als mythischer Weiser, als Hofgeist oder eben auch als Zar. In seinen Aufzeichnungen seiner Gespräche mit der Zarin finden wir zudem häufig Aussagen wie »si j'étais souverain «, »si j'étais roi«, »si j'étais sultan«, »me voilà couronné par les mains de Votre Majesté Impériale... ${ }^{24}$ So »regierte« Diderot tatsächlich öfters das Zarenreich auf dem Papier in einer Mischform aus naiver Utopie und politischem Realismus, freier Assoziation und annotiertem Gesetzestext, versteckt hinter scheinbar spontanen Gedanken oder Gesprächen. Dass Katharina Diderots »brillante Gedanken« nicht in die Tat umsetzte, war den Zeitgenossen bereits bekannt. Sein tatsächliches politisches Engagement, seine Einsichten und seine Vorschläge blieben der Öffentlichkeit sehr lange verborgen, da die Mehrzahl seiner Texte erst im neunzehnten und zwanzigsten Jahrhundert erschienen sind. ${ }^{25}$ Diderot hatte zwar seinerzeit sowohl das Gesetzbuch der Zarin als auch ihre Kommission, die sie für die Anfertigung des Nakaz gegründet hatte, um scheinbar im Habermas'schen Sinne der deliberativen Demokratie Kommunikation unter den Bürgern zu fördern, als Potemkin'sches Dorf in seinen Observations sur le Nakaz demaskiert, doch wurden diese erst 1920 publiziert. Damit weiß Sacher-Masoch davon noch nichts, lässt seinen Diderot den Affen spielen und kritisiert die pseudo-demokratische Lage in Russland in mehreren Zitaten der Zarin, wie zum Beispiel dem folgenden:

Man läßt einige knuten, andere schickt man nach Sibirien und die Vornehmsten auf das Schafott. Es ist pikant, einen Mann, mit dem man heute noch verbindliche Phrasen tauscht, morgen den Kopf auf den Block legen zu sehen...Nun ich finde es pikant, besonders, wenn ich denke, dass es nur von mir abhängt, diese Menschen, die da in Todesangst vergehen, zu begnadigen, daß ich, ich allein es bin, die sie sterben lässt. ${ }^{26}$

24 Siehe hierzu Denis Diderot: Mélanges philosophiques, historiques, etc., pour Catherine II, in: Euvres. Politique, hg. von Laurent Versini, Paris 1995, Bd. 3, S. 229, S. 203, S. 361, S. 255, S. 323, S. 240, S. 406f., S. 327f. und S. 259.

25 Diderots interessante Rezeptionsgeschichte beinhaltet Übersetzungen von seinen damals noch nicht publizierten Texten aus der Correspondance littéraire, die von Schiller und Goethe angefertigt wurden, den Verkauf vieler unveröffentlichter Manuskripte an Katharina die Große (Fonds de St. Petersbourg), sowie die abenteuerliche Entdeckung von noch unbekannten Manuskripten in einem Schloss in der Normandie nach dem Zweiten Weltkrieg (Fonds Vandeul). Siehe hierzu Denzel de Tirado: Biographische Fiktionen (wie Anm. 1), S. 35-37 und S. 92-94.

26 Sacher-Masoch: Diderot in Sankt Petersburg (wie Anm. 11), S. 24. 
So ist Katharina in Sacher-Masochs Geschichte von dem sprechenden Affen am Anfang zwar durchaus begeistert, dann aber bald gelangweilt, weil er keine Kunststücke machen kann. Sie vermisst ihre Gespräche mit dem französischen Philosophen über Platon. Diderot ist als »Affe" für die Zarin uninteressant, weil er in dieser Rolle kein Garant für eine scheinbar deliberative Demokratie mehr sein kann. Als sie erfährt, dass Diderot der »sprechende Affe« ist und ausgestopft werden soll, lacht sie nur und versucht keineswegs, ihn zu retten. Damit stellen sich die »verbindlichen Phrasen", die sie mit Diderot getauscht hat, als vollkommen bedeutungslose und leere Floskeln heraus, und sie ist bereit, ihn tatenlos sterben zu lassen.

Dies trifft im metaphorischen Sinne auch auf den realen Dialog zwischen Diderot und Katharina zu. Wie sein Vorgänger Platon scheitert auch Diderot daran, die Philosophie auf den Thron zu bringen. In einem Brief an Friedrich Melchior Grimm zeigt die Zarin ganz deutlich den Unterschied zwischen Wort und Tat und zwischen Papier und »menschlicher Haut« auf:

\begin{abstract}
Alors lui parlant franchement, je lui dis: >Monsieur Diderot, j'ai entendu avec grand plaisir tout ce que votre brillant esprit vous a inspiré; mais avec tous vos grands principes, que je comprends très bien, on ferait de beaux livres et de mauvaise besogne. Vous oubliez dans tous vos plans de réforme la différence de nos deux positions: vous, vous ne travaillez que sur le papier, qui souffre tout; il est tout uni, souple et n'oppose d'obstacles ni à votre imagination, ni à votre plume; tandis que moi, pauvre Impératrice, je travaille sur la peau humaine qui est autrement irritable et chatouilleuse. $<^{27}$
\end{abstract}

So scheitert Diderots »Konversationspolitik« und seine Hoffnung, dass nur er die Zarin aufklären könne, sowohl in Wirklichkeit als auch in Sacher-Masochs Fiktion. Die Zarin erweist sich in beiden Fällen als überheblich und uneinsichtig, sieht Diderot als einen nicht ernstzunehmenden politischen Ratgeber, und ein real demokratischer deliberativer Prozess findet nie statt.

Doch selbst wenn diese Gespräche mit Katharina niemals genau in dieser Form stattgefunden haben sollten, kann man doch von »deliberativer Demokratie« sprechen. Wie Markus Spörndli in seiner Analyse dieses Konzepts beobachtet, erklären einige »nicht-kommunikative

27 Katharina die Große, zit. nach Henry Vallotton: Catherine II et ses correspondants Voltaire, Diderot, Grimm et J.J. Rousseau, in: La Revue des deux Mondes 13 (1954), S. 659-674, hier S. 666. 
Theoretiker« wie Cass Sunstein und John Rawls, dass Deliberation »nicht unbedingt einen Dialog « beinhalten müsse, sondern auch durch vernünftige Abwägungen einer einzelnen Person zustande kommen könne, da »das Postulat der Unparteilichkeit« dann bereits erfüllt sei, wenn sich eine argumentierende Person "fiktiv in die Lage aller anderen betroffenen Leute versetze ${ }^{28}$

Während Leopold von Sacher-Masoch seinen Diderot im neunzehnten Jahrhundert als einen nicht ernst zu nehmenden, unredlichen und naiven politischen Berater stilisierte, findet in Peter Pranges Roman Die Philosophin durchaus eine Form von deliberativer Demokratie statt.

Wie in Sacher-Masochs Geschichte spielen auch hier zwei Frauen eine wichtige Rolle. Doch während Diderot in Diderot in Sankt Petersburg den Reizen und Verführungskünsten der beiden mächtigen Frauen zum Opfer fällt, sich von ihnen buchstäblich "zum Affen machen« lässt, gedemütigt wird und erfolglos nach Frankreich zurückkehrt, sind es bei Peter Prange gerade die Frauen, die sein Lebenswerk vor der Zensur und dem Verbot retten und ihn so zum Wegbereiter der Aufklärung und der Demokratie werden lassen. Bei Sacher-Masoch wird Diderots Geist von seinem sexuellen Begehren und seinem Spaß an Intrigen vollkommen ausgeschaltet, und seine Leidenschaft für die Frauen und deren Unterhaltung wird bestraft. In Peter Pranges Roman stellt Diderot sein Werk zwar über seine Gefühle, doch ist es auch gerade sein großes Herz, die Liebe und die Sexualität in diesem historischen Liebesroman, die seinen Erfolg gewährleisten.

Peter Pranges Die Philosophin (2003) und die Verbindung von Konversationspolitik und Sexualität

Peter Pranges belletristischer Historienroman konzentriert sich auf die Geschichte der Enzyklopädie und Diderots bekannte Brieffreundin Sophie Volland. Die Enzyklopädie wird als hochpolitisches und äußerst brisantes Buchprojekt gefeiert und als »das Buch der Bücher, ein Buch wie die Bibel, ein wirklich neues Testament für eine neue Zeit« bezeichnet. Dieses »neue Testament« hebt nicht nur »die Welt aus den Angeln«,

28 Spörndli: Diskurs und Entscheidung (wie Anm. 16), S. 14. 
sondern zeigt auch, »wie das Leben sein kann und sein soll ${ }_{.}^{29}$ Prange weist in mehreren Beispielen darauf hin, dass Diderots Lebenswerk die Menschen von Aberglauben und dem Dogma der Vergangenheit befreit und dadurch freier und glücklicher gemacht hat. Diderots Enzyklopädie, die das Wissen der Menschheit verbreitet und den Menschen zugänglich macht, verändert und verbessert die Kultur und öffnet neue Horizonte für Herrscher und Volk. ${ }^{30}$ Hier hebt Prange besonders Diderots Idee einer kollektiven Autorschaft hervor und stellt die Tatsache heraus, dass Diderot auch Handwerker einlud, um an dem klassenübergreifenden Sammelprojekt menschlichen Wissens mitzuschreiben. Dadurch schafft Diderot laut Prange eine Vorform von öffentlicher Beratung, eine Art von öffentlichem Diskurs, an dem die Bürger teilhaben können. Die Enzyklopädie wird dadurch zu einer Art praktischem input-orientierten Demokratiemodell, das als Plattform eines politischen Diskurses und der politischen Willensbildung der Bürger dient. Doch ein machtfreier Diskurs ist im achtzehnten Jahrhundert noch nicht gegeben und die Enzyklopädie hat viele Gegner. Dementsprechend wird Diderot als mutiger Revolutionär und sein Werk als »Sturmgeschütz der Vernunft, Armada der Philosophie« und »Kriegsmaschine der Aufklärung « gefeiert. ${ }^{31}$ Stets kriegerischen Allegorien folgend, stellt Prange seine Figuren nach manichäischer Manier einander gegenüber und platziert auf der einen Seite die innovativen und couragierten Aufklärer und auf der anderen Seite die mächtigen Gegner der Enzyklopädie.

In dieser dualistischen Gegenüberstellung kommt der Romanheldin, Sophie Volland, eine besondere Rolle zu. Sie wird als eine Figur der modernen Gesellschaft stilisiert, in der man das Recht, politisch zu verstehen, zu urteilen und mitzusprechen, jederzeit erlangen kann. Den Genreregeln des kritischen Frauenromans und des historischen Liebesromans folgend, überwindet Sophie Volland gesellschaftliche Zwänge und Standesgrenzen, kommt problemlos mit den wichtigsten historischen Figuren der Epoche in Kontakt, zieht im Hintergrund die Fäden und macht sich so unabdingbar für den Erfolg der Enzyklopädie und der Aufklärung. Prange entwirft hier eine vollkommen fiktionalisierte typische Heldin eines historischen Trivialromans und

29 Siehe Peter Prange: Die Philosophin, München 2003, S. 63.

30 Ebd., S. 97.

31 Ebd., S. 43. 
lässt geschichtliche Fakten über die reale Sophie Volland außer Acht. ${ }^{32}$ So konstruiert er Sophie als Opfer einer abergläubischen und primitiven Gesellschaft. Sie wächst als Waise in einem Kloster auf, weil ihre Mutter als Hexe verbrannt wurde. In der Lebensgeschichte Sophies können wir so die Entwicklung vom »dunklen Mittelalter« zur Aufklärung beobachten, die sich am Ende kaum noch von unserer modernen Gesellschaft unterscheidet. Prange macht das berühmte Café Procope, das tatsächlich Treffpunkt der Klassen- und Ständeunterschiede überwindenden Gesellschaft war, in seinem Roman zum Hauptspielort seiner Geschichte. Das Kaffeehaus wird hier - gemäß den von Habermas im Strukturwandel der Öffentlichkeit beschriebenen Kriterien - als liberales und offenes Modell bürgerlicher Öffentlichkeit zelebriert. Die hier »zum Publikum versammelten Privatleute« räsonieren, diskutieren und verhandeln gemeinsame Belange. Hier arbeitet Sophie als Kellnerin, was der jungen Frau, die später zur Vorleserin der Madame de Pompadour und zur Geliebten des höchsten Zensors des Königs, Chrétien-Guillaume de Lamoignon de Malesherbes, aufsteigen sollte, erlaubt, an dieser eigentlich nur Männern und gebildeten Bürgern vorbehaltenen Öffentlichkeit zu partizipieren. Hier lernt sie sowohl Diderot als auch seinen Antagonisten, den Polizeioffizier Sartine, den sie später heiratet, kennen, denn das Procope war voll von »Spitzeln der Polizei, die sich im Café Procope eingenistet hatten wie Flöhe in einer alten Perücke«. ${ }^{33}$ Dank der Vermittlerfigur Sophie Volland, die nicht nur kinderleicht Klassenunterschiede überwinden kann, sondern auch spielerisch politische Überzeugungen mächtiger Männer und Frauen ändern kann, hat Diderot schließlich auch Freunde in hohen Positionen, die ihn in seiner bildungspolitischen Aufgabe unterstützen. Prange imaginiert Sophie Volland von Anfang an als Diderots Muse und Inspiration für dessen Sozialkritik. So schreibt er, dass Diderot die Kellnerin Sophie im Café Procope getroffen und sie ihn dort inspiriert habe, ein wunderschönes Liebesmärchen mit dem Titel »Die Prinzessin Mizorza und der Sultan Mongagul« für sie zu schreiben. Nach der Lektüre des Manuskripts und einer wunderbaren Liebesnacht mit Diderot - ein äußerst beliebter Topos in biographischen Fiktionen über den

\footnotetext{
32 Für mehr Information zu Diderots berühmter Brieffreundin, siehe Heidi Denzel de Tirado: Diderot als Briefeschreiber und der Traum von SMS und E-mail, in: Gesellschaft für kritische Philosophie 4 (2013), S. 89-100.

33 Prange: Die Philosophin (wie Anm. 29), S. 56.
} 
impulsiven Aufklärer, besonders in den französischen Werken - wird das Liebesglück sofort von Diderots eifersüchtiger Frau zerstört. Jahre später findet Sophie das für sie geschriebene Märchen in dem verbotenen Buch Die geschwätzigen Kleinode wieder, das sie auf dem Nachttisch ihres Ehemanns Antoine Sartine, Polizeioffizier und Spion des Königs, findet. Verwirrt darüber, dass aus dem romantischen Liebesmärchen bitterböse Gesellschaftskritik geworden ist, verrät sie ihrem Mann den Namen des anonymen Autors und trägt damit ungewollt zu Diderots Verhaftung bei. Durch diese Änderung der Entstehungsgeschichte der Bijoux Indiscrets, die allgemein als Diderots politischste Satire und schärfste Kritik des Despotismus angesehen werden, entreißt Prange dem Autor sein politisches Anliegen und Engagement und reduziert Diderots Kritik auf eine persönliche Frustration eines verletzten Liebhabers und führt damit Diderots komplexe sexualpolitische Allegorie kunstlos biographistisch auf dessen Leben zurück. Dem Vorbild der »Konversationspolitik" der Les Bijoux Indiscrets folgend webt Prange dann aber zeitlos relevante philosophische, ethische und politische Fragen in Gespräche ein, die aufkommen, »wenn Menschen miteinander streiten, aneinander vorbeireden, sich umgarnen oder miteinander schlafen ${ }^{34}$.

Nach einer langen Schilderung von Diderots Aufenthalt im Gefängnis, in der Prange den couragierten, anständigen Diderot mit dem als Opportunisten und Feigling beschriebenen Rousseau kontrastiert, verlässt Sophie ihren Ehemann und trifft wieder auf Diderot im Café Procope. In Pranges fiktiver klassenlosen Gesellschaft erhält sie daraufhin mühelos ihr Mitspracherecht und steigt als Diderots Geliebte zur Autorin und Redakteurin der radikalsten politischen Artikel der Enzyklopädie auf. Ihre Erweiterung des Beitrags von Abbé des Prades führt schließlich zum Verbot der Enzyklopädie und Diderot verbietet ihr daraufhin die Mitarbeit an der Enzyklopädie. So endet das philosophische Liebesglück erneut, dieses Mal, weil Diderot sein Werk über seine Gefühle stellt.

Doch Sophie gibt nicht auf und beginnt jetzt erst recht, politisch und philosophisch aktiv zu werden. Unermüdlich vermittelt sie von nun an zwischen den Fronten der Aufklärung und ihrer Gegner. Als Zofe im Haus des Bruders der Madame de Pompadour bittet sie die einflussreiche Mätresse des Königs, sich für die Veröffentlichungserlaubnis der

${ }^{34}$ Katie Trumpener: The Politics of Conversation (wie Anm. 10), S. 225. 
Enzyklopädie einzusetzen. Prange benutzt hier - wie viele von Diderots Erzählungen - Sexualität als »politisches Motiv«, um die Unschlüssigkeit und Flatterhaftigkeit von Sexualität und politischer Organisation herauszustellen. ${ }^{35}$ Erneut dreht Prange Prinzipien von Ursache und Effekt um, indem er behauptet, dass sich die Mätresse des Königs von Diderots Les Bijoux Indiscrets inspiriert gefühlt habe, ein kleines Bordell im Hirschpark von Versailles für den König zu eröffnen. Damit wird dieses allgemein als Schlüsselroman verstandene Werk, in dem Mongagul deutlich als Louis XV und Mirzoza augenscheinlich als Madame de Pompadour erkennbar sind - bei Prange zur Inspiration für Madame de Pompadour, den König sexuell bei Laune zu halten, um politisch Einfluss auf ihn nehmen zu können. Die Pompadour ist Diderot deswegen äußerst dankbar für diese »sexualpolitische Idee«. Obwohl Sophie das Angebot aus Liebe zu Diderot ausschlägt, eine Dame im Pavillon des Königs im Hirschpark zu werden, verwendet sich die Pompadour für die Enzyklopädie. Und sie tut dies nach Diderot'scher Formel in scheinbar lapidaren Alltagsgesprächen. Als der König nach einem enttäuschenden Jagdausflug wissen will, warum seine Büchse eine Ladehemmung hatte, wirft "Mirzoza" beiläufig ein, dass sie gerne wissen würde, wie ihr Gesichtspuder seine Wirkung entfalte. Nach dem vermeintlich unschuldig formulierten Wunsch, dass es doch nützlich wäre, "wenn man solche Dinge irgendwo nachschlagen könnte«, lässt sie die veröffentlichten Bände der Enzyklopädie hereinbringen, der König ist von den Erklärungen der Enzyklopädie begeistert und hebt das Verbot auf. Prange schildert daraufhin die gesamte Geschichte der Enzyklopädie, die von Zensur, Verboten und Aufhebungen von Verboten geprägt ist, und verbindet weiterhin Sophies Schicksal und das Wohlwollen der Pompadour mit dem Werdegang des Buches.

Am Ende ist es Sophie, die den Erfolg der Enzyklopädie durch die richtigen Entscheidungen gewährleistet. Da sie als Mätresse des Zensors Malesherbes diesen zu einer Zeit, in der Diderots »Feinde alle Macht im Staat erobert haben«, dazu gedrängt hatte, sein Amt als oberster Zensor aufzugeben, bringt sie erneut ungewollt das Gelingen der Enzyklopädie in Gefahr. Doch auf ihrem Totenbett macht die Pompadour Sophie bewusst, dass es »ihre Pflicht [sei], Diderot zu helfen« und dass sie »die Einzige [sei], die ihn schützen kann. Ihn und die Enzyklopä-

35 Vgl. Valérie André: Diderot. Contes politiques et politique du conte, in: Féeries. Études sur le conte merveilleux (XVIIe-XIXe siècle) 3 (2006), S. 137-157. 
die ${ }^{36}$ Bei Prange sind es damit also zwei Frauen, die verstehen, dass man eine deliberative Demokratie letztendlich nur durch Kompromisse, Zugeständnisse und eine Politik der argumentativen Abwägung erreichen kann: »Sie musste die Vollkommenheit des Werkes zerstören, Worte der Wahrheit und der Vernunft für immer auslöschen, um das Ganze vor der Vernichtung zu bewahren. ${ }^{37}$ So interpretiert Prange Le Bretons perfide Verstümmelung und Autozensur der Enzyklopädie zwar als notwendige Entscheidung, um dieses wichtige Buch veröffentlichen zu können, traut ihm aber eine so mutige Handlung nicht zu. Da sich Le Breton als zu feige erweist, ist es Sophie, die Diderots Werk am Ende »verfälscht, verstümmelt, kastriert« und ihn damit »betrogen, gedemütigt, zerstört « zurücklässt. ${ }^{38}$ Diderot will seine Herausgeberschaft daraufhin aufgeben, doch Malesherbes macht ihm die wegweisende Bedeutung seines Werkes bewusst: »Sie haben nicht nur das Wissen darüber gesammelt, wie man die Welt verbessern kann, sondern mit der Enzyklopädie auch ein Programm zur praktischen Verwirklichung entworfen.« Diese Verbindung demokratietheoretischer Konzepte und deren praktischer Umsetzung werden allgemein als wesentliches Kennzeichen der deliberativen Demokratie bezeichnet, und so erscheint das Enzyklopädieprojekt wie ein Modell für beteiligungszentrierte Demokratie. Überzeugt von dem Argument, dass Zensur, »wenn es eine Zensur geben muss, aus welchen Gründen auch immer", am besten durch »einen Gegner der Zensur ausgeübt wird«, vergibt Diderot seiner Sophie am Ende mit den Worten: »Damit wir gemeinsam zu Ende bringen, was wir angefangen haben «. ${ }^{39}$

Diderot und Sophie erscheinen in diesem Roman als Wegbereiter der Moderne und »Zeitgenossen der Gegenwart«. Durch den ständigen Dialog wird bei Prange nicht nur das politische Bewusstsein der Eliten verändert, sondern auch die politische Willensbildung der Massen. Während Sophie den obersten Zensor und die Pompadour in ihren Gesprächen von der Wichtigkeit der Enzyklopädie überzeugen kann, ist es Diderot, der die einfachen Leute zum Denken veranlasst. Hier erscheint er hauptsächlich als aktiver Zuhörer, ermöglicht ihnen dadurch die Zugehörigkeit zu der neuen Öffentlichkeit und gibt ihnen da-

36 Prange: Die Philosophin (wie Anm. 29), S. 485.

37 Ebd., S. 475.

38 Ebd., S. 504f.

39 Ebd., S. 517. 
durch eine Stimme. Während sich Prange am Anfang des Buches auf die Schilderung von Diskursen entstehender politischer und sozialer Bewegungen konzentriert, endet er seinen Roman mit Beschreibungen der großen politischen Umwälzungen des achtzehnten Jahrhunderts. So schildert er den Einfluss der Enzyklopädie auf politische Aktionen wie den Mordanschlag auf den König, den Marsch der Pariser Frauen nach Versailles - an dem er Sophie natürlich 1789 aktiv teilnehmen lässt - und schließlich die Revolution. Er erklärt wie der dritte Stand die Macht erobert hatte und wie die feudalen Standesrechte von nun an durch angeborene, unantastbare und unveräußerliche Menschenrechte ersetzt worden seien, um die Freiheit der Bürger gegen die Willkür des Staates zu schützen. Er gibt der Enzyklopädie damit eine wichtige Rolle für die politische Meinungsbildung und sieht sie als Teil einer sich langsam bildenden politischen Öffentlichkeit. Obwohl das Leben nach der Revolution, das Prange im Epilog aufzeigt, wie eine klare Widerspiegelung der Gegenwart wirkt, zeigt sich Sophie doch revolutionskritisch. Es ist der zum Tode verurteilte Malesherbes, der die Philosophin - und die Leser - in einem letzten Gespräch von der Wichtigkeit der Aufklärung überzeugt, die er als eine »immer wieder neue Aufgabe« bezeichnet, an »der jede Generation weiterarbeiten muss«. Als Initiator dieser großen Aufgabe sieht er Diderot und dessen Lebenswerk: »Die Enzyklopädie hat für die Menschheit ein riesiges Tor aufgestoßen, jetzt liegt es nur an den Menschen, durch dieses Tor zu schreiten ${ }^{4}{ }^{40}$

Die Entstehung politischer Willensbildung und der Dialog mit der Nachwelt

In den beiden analysierten Texten erscheint Diderot als ein "Zeitgenosse der Zukunft«, der mit der Nachwelt plaudert, ein Phänomen, das sich allgemein in den fiktionalbiographischen Texten des zwanzigsten und einundzwanzigsten Jahrhunderts über Diderot als Hauptfigur zeigt: »je ne vous écris pas; mais je cause avec vous [gemeint ist der Leser] ${ }^{41}$ Prange folgt der von Jean-Pierre Seguin beschriebenen Formel einer »doppelten Orientierung« von fiktiven Zeitreisen bei Diderot, die sich

\footnotetext{
40 Ebd., S. 542.

41 Roger Kempf: Diderot et le roman ou le démon de la présence, Paris 1964, S. 35.
} 
einerseits »vers l'avenir, et c'est la science-fiction« richten und andererseits »vers le passé, et c'est la constitution de mythe «. ${ }^{42}$ Sacher-Masoch und Prange rezipieren Diderots zukunftsweisende Gedanken auf unterschiedliche Art. Sacher-Masoch entwirft Diderot und das Russland des achtzehnten Jahrhunderts als Relikte einer vollkommen anderen Zeit zum Kontrast seiner Gegenwart des neunzehnten Jahrhunderts, und Prange vergleicht das Frankreich der Aufklärung mit der Lebenswelt seiner Leser.

In beiden Geschichten gibt es eine Frau, die im politischen Zentrum steht und politische Macht besitzt und von einer weniger mächtigen Frau beraten wird. Bei Sacher-Masoch ist es die Gräfin Daschkoff, die Katharina die Große beeinflussen kann, und bei Prange ist es die Marquise de Pompadour, die sich von Sophie inspirieren lässt. Alle vier Frauen spielten tatsächlich eine wichtige Rolle in Diderots Leben, doch hing das Wohlwollen der Zarin und der Pompadour ihm gegenüber weder von Sophie Volland noch von der Gräfin Daschkoff ab. Obwohl beide Geschichten auf den ersten Blick hauptsächlich als Liebesgeschichten erscheinen, versteckt sich hinter ihnen - ganz auf Diderot'sche Art - durchaus scharfe Kritik am politischen System.

Beide Texte fingieren Orte der öffentlichen Debatte, die Habermas in Strukturwandel der Öffentlichkeit als Plattformen des öffentlichen Diskurses bezeichnet hat. Sacher-Masoch beginnt seine Geschichte in der Akademie der Wissenschaften und Prange in einem Kaffeehaus. Auffallend ist, dass sich diese Orte in den Fiktionen als allen zugängliche, offene Diskussionsforen ohne sozial-, bildungs- und geschlechtsspezifische Grenzen präsentieren und damit auf den ersten Blick dem Ideal einer bürgerlichen Öffentlichkeit entsprechen, denn in beiden Texten sind sowohl Bürger der unteren Klassen als auch Frauen erlaubt. Doch in beiden Fällen kann nicht gemeinsam räsonierend nach der Wahrheit gesucht werden. Bei Sacher-Masoch glaubt Lagetschnikoff, dass er aufgrund einer kritischen Frage getötet und ausgestopft werden könne, und Diderot wird aufgrund seiner leidenschaftlichen Reden von Sartine überwacht, festgenommen und in das Staatsgefängnis im Donjon von Vincennes gebracht. In beiden Texten werden Diskurse und Reden entstehender sozialpolitischer Bewegungen thematisiert. Während Diderots Genie und Einsichten in Russland keinerlei Spu-

${ }^{42}$ Jean-Pierre Seguin: Diderot, le discours et les choses. Essai de description du style d'un philosophe en 1750, Paris 1978, S. 177. 
ren hinterlassen, sieht Peter Prange in ihm den Autor des Buchs, das die Welt aus den Angeln gehoben und für immer verändert hat. Obwohl sich die deutsche Rezeption - sei es Diderot und das dunkle Ei, Leopold von Sacher-Masochs Novelle Diderot in Sankt Petersburg, Peter Pranges Roman Die Philosophin oder Michael Krügers Gedicht Diderots Katze - zwar nicht explizit auf Diderots politische Texte konzentriert, so thematisieren doch alle sein Interesse und seine Offenheit, sich auf andere Menschen - und Tiere - einzulassen. So zollt ihm die für den bescheidenen Philosophen so wichtige Nachwelt - »le grand juge qui nous jugera tous: la postérité « ${ }^{43}$ - im neunzehnten, zwanzigsten und einundzwanzigsten Jahrhundert genau die Ehre, die er sich immer erhofft hat. Diderot, dieser »homme sans vie« - wie Bonnet ihn in Anlehnung an den »homme sans ombre« der Romantik bezeichnet hat - wird als »grand homme« noch aus seinem Grab gehört. In Deutschland wird er außerdem genauso in Szene gesetzt, wie es seinem Ideal entspricht; nicht als Hauptprotagonist auf der politischen Bühne, sondern als »homme de génie« im Dialog »au parterre«. ${ }^{4}$

43 Denis Diderot: Lettre à Falconet du 15 Février, in : Correspondance, hg. von Georges Roth und Jean Varloot, Paris 1955-70. Bd. 5, S. 58-100, hier S. 75.

44 Ich beziehe mich hier auf zwei berühmte Zitate von Diderot. Das erste stammt aus dem Essai sur les règnes de Claude et de Néron, Paris 1779, S. 297: "[O]n ne pense, on ne parle avec force que du fond de son tombeau: c'est là qu'il faut se placer, c'est de là qu'il faut s'adresser aux hommes. "Und das zweite Zitat kommt aus dem Paradoxe sur le comédien: "[D]ans la grande comédie, la comédie du monde [...] toutes les âmes chaudes occupent le théâtre; tous les hommes de génie sont au parterre."Zit. nach Jean-Claude Bonnet: La Naissance du Panthéon. Essai sur le culte des grands hommes, Paris 1998, S. 171. 